\title{
A Importância da Comunicação em Saúde
}

\author{
Paulo Sérgio Nunes dos Santos ${ }^{1}$ \\ António Maria Salvado Coxito Granado ${ }^{2}$ \\ Henrique Girão ${ }^{1}$ \\ ${ }^{1}$ Universidade de Coimbra, Portugal: E-Mails: psnsantos@gmail.com; hmgirao@fmed.uc.pt \\ ${ }^{2}$ Universidade Nova de Lisboa, Portugal; E-Mail: agranado@fcsh.unl.pt
}

\section{Resumo}

O propósito deste artigo é demonstrar, de forma sucinta, os caminhos a trilhar pela comunicação de ciência, mais especificamente pela comunicação em saúde, em termos institucionais e no contexto de uma Escola Médica. Entendese que esta área tem sido desvalorizada, mas assume, atualmente, uma enorme preponderância no sentido de uma responsabilização por parte da sociedade, dado que é o elo entre comunicação científica e o público não especializado. Assim, seguindo algumas tendências recentes, propõe-se que uma Escola Médica não deve descurar esta área de estudo, mas antes reforçar a aposta, assentando-a em quarto valências fundamentais - formação, educação cívica, promoção e divulgação, e inovação e investigação, - formando melhores profissionais e aproximando a sociedade daquilo que são as suas áreas de investigação.

Palavras-chave: comunicação de ciência; comunicação institucional; comunicação em saúde; Escola Médica; formação.

\begin{abstract}
The purpose of this article is to demonstrate, briefly, the paths to be taken by science communication, more specifically by health communication, in institutional terms and in the context of a Medical school. It is understood that this area has been devalued, but it presently takes on an enormous preponderance in the sense of a responsibility on the part of society, since it is the link between the scientific community and the non-specialized public. Thus, following some recent trends, it is proposed that a Medical School should not neglect this area of study, but rather reinforce the bet, based on four fundamental values- training, civic education, promotion and outreach, and innovation and research-, forming better professionals and bringing society closer to the research made.
\end{abstract}

Keywords: formation; health communication; institutional communication; Medical School; science communication.

\section{Introdução}

O ser humano tem comunicado, provavelmente, desde longa data. Olhando, por exemplo, para as pinturas rupestres, algumas com cerca de 40 mil anos (Gombrich, 2013), pode-se perceber o impulso do Homem em transmitir aos outros aquilo que viu, ouviu, experienciou. Comunicar ser-lhe-á, então, um ato inerente. E comunicar ciência surge como algo essencial, na medida em que os cientistas são, embora por vezes não parecendo aos olhos do público, seres humanos, que falam e escrevem (Montgomery, 2002). Para o mesmo autor, não pode haver divisões entre fazer ciência e comunicar essa mesma ciência, surgindo assim a habilidade de um cientista em falar e escrever sobre aquilo que faz como fator determinante da validação e importância da sua pesquisa e das suas descobertas. Porém, na opinião de (Greenwood \& Riordan, 2001), essa fusão não tem um cariz de obrigatoriedade, sendo que o papel do cientista enquanto comunicador de ciência deve antes resultar de um apelo pessoal a prestar um serviço público.

Knorr-Cetina acrescenta que a comunicação de ciência é também um mecanismo inerente de perpetuação: 
“a comunicação é dita ser intrínseca à ciência pelo facto de a ciência moderna ser um empreendimento colectivo que depende de os resultados obtidos por cientistas individuais serem retomados por outros cientistas que se fundam neles e os desenvolvem. A ciência projecta-se a si mesma no futuro através da comunicação. Uma ciência privada é tão impensável como uma linguagem privada." (Knorr-Cetina, 1999, p. 378).

Conseguimos então perceber que a ciência tem um alcance sem precedentes na vida de qualquer cidadão (Leshner, 2003). Qualquer vertente da sua existência é determinada por esta relação nem sempre visível (Barbeiro, 2007). É, por isso, natural o surgimento de diferentes modelos e formas de comunicar ciência, cujo objetivo final é uma participação de todos, sem fronteiras, no processo e na vida científica.

"O exercício da cidadania e a eficácia do processo democrático dependem largamente de um melhor conhecimento, por parte dos cidadãos, de temas relacionados com a ciência e a tecnologia, que muitas vezes estão na base de diversas decisões políticas" (Araújo, Bettencourt-Dias, \& Coutinho, 2006, p. 1).

\section{Ciência na Comunicação}

Definir um conceito como o de ciência, que adquire um cariz quase pessoal, é uma tarefa que se mantém ainda em aberto até aos dias de hoje. A ciência é um mundo complexo, a começar pela sua abrangência de domínios e áreas do saber; dessa forma, o seu significado não pode ser simples nem, consequentemente, resumido em poucas palavras (Shamos, 1995).

Quando se fala em comunicar ciência, o foco passa de encontrar uma definição menos ou mais complexa para se conseguir compreender o que se entende por ciência, ou seja, até onde se traça a fronteira daquilo que é comunicado como ciência. Uma das definições mais citadas na literatura data de 1986. Friedman, Dunwoody e Rogers (como citado em Weigold 2001) propuseram que ciência é um conceito que abrange, em si próprio, múltiplas ciências, das ciências naturais e exatas às sociais e comportamentais, entrando ainda em áreas mais aplicadas: tecnologia, engenharia, ciências ambientais ou medicina.

Mais recentemente, (Burns, O'Connor, \& Stocklmayer, 2003) sublinharam essa necessidade de clarificação, que assume contornos mais importantes quando as definições de ciência variam entre profissionais conforme as áreas em que trabalham; é frequente a ciência terminar quando o assunto é medicina, passando então para o domínio da saúde (Weigold, 2001).

\section{Comunicação de Ciência}

Quando se debate comunicação de ciência, está em causa uma terminologia cujo uso se convencionou nas duas últimas décadas para descrever o seu campo de estudo e as teorias a ele associadas (Bucchi \& Trench, 2016). Os dois autores utilizam um exemplo, a recente utilização da expressão "ciência na sociedade" como um quase sinónimo para comunicação de ciência, o que faz com que haja alguma ambiguidade na nomenclatura de uma ciência ainda em desenvolvimento e uma consequente diversidade de termos para as mesmas práticas e os mesmos significados (Bucchi \& Trench, 2016).

Para efeitos de comunicação de ciência, a ciência deve incluir tudo o que Friedman et al. (como citado em Weigold 2001) e Burns et al. (2003) consideram, ou seja, toda e qualquer área onde haja avanços científicos, fale-se de biologia, medicina ou história - as ciências da comunicação de ciência (Fischhoff, 2013). Ao estabelecer o que a ciência contem, deve avançar-se no sentido de clarificar o que se entende por comunicá-la. Também neste campo os conceitos são variados.

Pode considerar-se a comunicação de ciência como o conjunto de atividades efetuadas por comunicadores profissionais, entre eles jornalistas e cientistas (Treise \& Weigold, 2002). De uma forma mais complexa, Harwood e Schibeci (2011, como citado em Palmer e Schibeci: 2012) conceptualizam-na como um processo cujo propósito é partilhar conhecimento através da interação entre as partes envolvidas. Essa partilha vai depender de dois fatores: primeiro, a relação que se estabelece entre o emissor e o recetor, e segundo, o objetivo que levou à comunicação do conhecimento. 
Fischhoff (2013) refere que, tratando-se a comunicação de ciência de um processo que pretende dotar o seu público com ferramentas decisórias, deve-se começar primeiro por ouvir os interessados. Só assim, conhecendo a sua realidade, o que já sabem e percebendo os problemas com que se deparam, é possível determinar a informação, ou a ciência, de que necessitam. Após este conjunto de ações, quem comunica ciência deve então criar métodos para atingir os resultados propostos, que serão posteriormente avaliados para que se determine a necessidade de repetição do processo (Fischhoff, 2013).

Retornando a Burns et al. (2003), a comunicação de ciência surge do uso de diferentes tipos de recursos (atividades, capacidades, media, diálogo), cuja finalidade é promover uma ou mais reações em relação à ciência. Para os autores, essas reações são definidas através do acrónimo AEIOU: tomada de consciência, satisfação, interesse, formulação de opinião e compreensão alargada sobre o que é, então, a ciência (em inglês, 'Awareness, Enjoyment, Interest, Opinionforming and Understanding').

António Granado e José Vítor Malheiros, antigos jornalistas de ciência no Público, salientam a independência da comunicação de ciência em função de determinados parâmetros:

Reúnem-se sob a designação de "comunicação de ciência" todas as actividades que visam comunicar o saber científico, os resultados da investigação científica ou informação sobre o contexto em que esta é feita, em todas as áreas, independentemente dos públicos considerados (que pode ser um grupo restrito dentro da comunidade científica, toda a comunidade científica, as crianças em idade pré-escolar, os deputados do Parlamento ou os habitantes de uma dada aldeia), do contexto em que a comunicação tem lugar, das ferramentas usadas ou do objectivo dessa comunicação. (Granado \& Malheiros, 2015, p. 16)

Para além de toda a possibilidade de públicos, há também que considerar que existe uma variedade considerável de profissionais que se podem enquadrar na categoria de comunicadores de ciência

Ainda que a expressão "comunicação" esteja (erradamente) associada a comunicação institucional ou mesmo a manipulação e propaganda, a comunicação de ciência inclui a actividade dos profissionais dos museus, centros de ciência, jardins botânicos, zoológicos e aquários; dos profissionais de gabinetes de comunicação de laboratórios e universidades; dos jornalistas que trabalham em ciência; dos autores de documentários, de software ou de websites dedicados à ciência; dos autores de newsletters, de programas de televisão ou de rádio sobre ciência; dos ilustradores, designers, infográficos e fotógrafos que trabalham em ciência; dos autores de livros de divulgação científica; dos organizadores de congressos, de festivais ou de feiras de ciência; dos conferencistas profissionais ou entertainers que trabalham na área da ciência; dos tradutores científicos; dos professores e formadores que formam toda esta gente. (Granado \& Malheiros, 2015, p. 16).

De forma resumida, "'Comunicação de ciência' é a expressão mais genérica, mais vaga, mais inclusiva, mais técnica e mais fria que se pode usar neste contexto e cobre todas as actividades onde a ciência se cruza com a comunicação" (Granado \& Malheiros, 2015).

\section{A Comunicação de Ciência Institucional}

A expressão de António Granado e José Vítor Malheiros pode parecer negativa para o próprio conceito de comunicação de ciência, mas, analisada mais profundamente, trata-se de uma forma curta de concetualizar todo um mundo e uma área de estudo que apenas agora começam a ganhar preponderância em contextos académicos e sociais. Há uma linha de pensamento, nas universidades e a uma escala global, assente numa noção de que a sociedade sabe e compreende o trabalho por elas desenvolvido. Se em termos educativos isso pode ter algum fundamento, pela aparente interligação formação-emprego, entender o que se faz ao nível científico é mais complicado para quem não é especialista na matéria. A juntar a isto, é ainda necessário assegurar que a comunicação entre cientistas e instituições e o público em geral seja o mais clara possível.

Muitas vezes, o problema pode não residir na complexidade da investigação científica desenvolvida, mas antes na falta de explicações acerca da aplicação dos seus resultados no comum quotidiano e também dos passos futuros a tomar, bem como do tempo necessário para os alcançar e de possíveis obstáculos que possam surgir. A ciência é, 
frequentemente, vista pelos cidadãos como uma área cujas conclusões têm de ter um uso imediato, inquestionável e economicamente benéfico (Brownell, Price, \& Steinman, 2013a).

Um dos casos mais mediáticos da comunicação de ciência a um patamar institucional é o da 'National Aeronautics and Space Administration', a agência do Governo Federal dos Estados Unidos comummente conhecida pelo seu acrónimo NASA. Fundada a 29 de julho de 1958, a partir da anterior estrutura, o 'National Advisory Committee for Aeronautics', a NASA teve, entre 1959 e 1963, uma parceria com a revista 'Life', que resultou em histórias sobre os astronautas norte-americanos, que preencheram páginas de 28 números da publicação. O objetivo era o de promover a necessidade dos avanços na área espacial, que englobava muitos outros campos científicos, através do acesso ao imaginário da sociedade, publicitando os astronautas como heróis, mas ao mesmo tempo pessoas comuns, como qualquer outro indivíduo. $O$ início da ligação entre as duas instituições teve lugar em setembro de 1959, com uma peça de 18 páginas que incluiu fotografias a cores do treino dos astronautas (Garber, 2013).

Alguns anos mais tarde, em 1967, dados de um estudo efetuado pela National Association of Science Writers, também nos Estados Unidos, mostraram que, em cerca de duas mil pessoas inquiridas, 83 por cento referiram querer compreender melhor o que se faz em termos de investigação científica, dado essa ser uma forma de melhorar tudo aquilo que rodeia a sociedade (Krieghbaum, 1970, como citado em Ribeiro 2016). Partindo do pressuposto que este número possa ser generalizado, ainda que num patamar mais baixo, perceciona-se, atualmente, um interesse considerável das pessoas pela ciência, principalmente quando incentivadas.

Assume-se então uma vertente mais tática, uma "comunicação estratégica de ciência", como refere Ricardina Magalhães (2015, p. 54): uma "estratégia que se baseie na descodificação das descobertas científicas para o público que não pertença a este meio".

Nesse contexto, e especificando um outro, o institucional, pode-se afirmar que é missão de uma Faculdade de Medicina, de uma Escola Médica, não só formar novos profissionais de saúde, mas também contribuir para uma sociedade com mais cultura e educação científicas, que possa perceber e encarar de uma forma mais esclarecida, com mais propriedade e, eventualmente, com mais esperança, os desafios atuais. $O$ sucesso de uma grande instituição passa, nos dias de hoje e em larga medida, pela visibilidade, credibilidade e impacto que tem aos olhos da sociedade.

Para tal, é necessário dar a conhecer o que é produzido nos laboratórios de investigação científica, abrindo-os à sociedade e levando a ciência às pessoas. Para conseguir atrair os melhores alunos e investigadores, é determinante assumir uma estratégia de divulgação da instituição forte, aguerrida, assertiva e impactante. Para chegar às pessoas, de uma forma eficiente e frutífera, há não só que desenvolver formas inovadoras e eficazes de comunicação, como também desenhar programas suficientemente atrativos que despertem a atenção e o interesse dos cidadãos.

Assim, esse caminho deve assentar em quatro pilares fundamentais, que devem orientar todo o esforço institucional de reconhecimento interno e externo do trabalho efetuado: formação, educação, promoção e divulgação, e investigação e inovação.

\section{Formação}

Um problema que se coloca frequentemente no relacionamento cientista-público é a dificuldade de comunicação, de fazer chegar, de uma forma simples e percetível, a mensagem científica (Nisbet \& Scheufele, 2009). Temas como as alterações climáticas (Somerville \& Hassol, 2011), a vacinação (Gross, 2009), ou a evolução (Miller, Scott, \& Okamoto, 2006) figuram entre os mais polémicos, pela quantidade de desinformação existente numa época de acesso fácil a conteúdos de veracidade duvidosa, mas também pelas implicações políticas e sociais de determinadas temáticas. Também no âmbito das apresentações em congressos científicos, ou aulas, uma postura, abordagem e atitude adequadas são determinantes para atrair a atenção e interesse da audiência. Por isso, é essencial treinar cientistas, incluindo médicos, a "usar", de uma forma proveitosa, os diversos meios de comunicação disponíveis, para transmitir a informação científica e tecnológica, nomeadamente novos avanços e descobertas que poderão beneficiar ou ser do interesse dos cidadãos. Dessa forma, devem ser criados processos de formação adequados, para melhorar a eficiência da transmissão da mensagem científica, quer entre pares, no âmbito de reuniões científicas, quer para o público em geral, diretamente ou através dos media. Um ponto crucial passa pela inclusão de unidades de comunicação de ciência na estrutura curricular de cursos conferentes de grau académico, por forma a despertar, desde cedo, o interesse e a importância de comunicar ciência, com vista à melhoria da qualidade do discurso entre cientistas e o público não especializado (Brownell, Price, \& Steinman, 2013a, 2013b). 


\section{Educação Cívica}

Outro dos pilares é determinado pela necessidade de um contributo para a promoção da educação e cultura científica da população ('outreach': "qualquer comunicação científica que, de forma direta, seja dirigida a um público não académico [Poliakoff \& Webb 2007, p. 244]). Percecionando a relevância, explicada anteriormente, da educação formal, no sentido formativo do termo, e de um contínuo investimento nessa área, é também importante que a aposta passe por um cada vez maior envolvimento com o público leigo, envolvimento esse que vai carecer de estratégias diferenciadas, que respeitem as audiências e os seus respetivos valores (Nisbet, 2010). A maior parte das metodologias e métodos utilizados atualmente ainda permanecem demasiado ligados a modelos de comunicação unilaterais, que pressupõem uma atitude subserviente e de total desconhecimento por parte da sociedade, pelo que, para se melhorarem as medidas implementadas e as suas taxas de participação, os cientistas devem aplicar o mesmo rigor científico que praticam no decorrer dos seus trabalhos, e não encarar a comunicação de ciência como algo marginal e de segundo plano (Varner, 2014). Nesse sentido, organizar fóruns deliberativos, cafés de ciência, ou mesmo reuniões abertas à sociedade em geral, podem representar, mesmo correndo o risco do seu atual excesso de utilização, contextos que se tornam importantes para o contacto direto com ativistas e outras partes interessadas (Nisbet, 2010). Ao mesmo tempo, é preciso adequar as estratégias usadas aos diversos públicos; o uso de redes sociais é hoje distinto consoante a faixa etária, e mesmo as temáticas abordadas podem não ser transversais a todas as gerações.

Uma sociedade com mais literacia científica é uma sociedade melhor preparada não só para enfrentar e ultrapassar as exigências e desafios que se colocam a cada momento às sociedades, facilitando a adoção de melhores práticas e comportamentos, mas também para perceber os desafios com que a ciência se depara a cada momento, possibilitando um papel mais interventivo e sustentado nas tomadas de decisão, no que respeita às decisões de estratégia científica (Gulbrandsen, 2016).

\section{Promoção e Divulgação}

Atualmente, para que uma instituição seja bem-sucedida tem de ter a devida visibilidade e reconhecimento por parte dos cidadãos. De facto, para atrair os melhores alunos e os melhores cientistas, as grandes instituições têm de ter uma atitude pró-ativa na divulgação e promoção das atividades por si desenvolvidas, quer ao nível do ensino quer da investigação (Shipman, 2014). Por exemplo, é necessário desenvolver estratégias fortes e assertivas de "apresentação" da Escola, promovendo, por exemplo, visitas aos espaços de investigação e criação de conhecimento. Uma adequada promoção passa também, e obrigatoriamente, por uma eficiente divulgação das atividades desenvolvidas no seio da instituição, nomeadamente as de natureza científica e tecnológica. Assim, há necessidade de se adotar critérios e práticas de divulgação do que de bom é produzido na Escola, e que poderá ter impacto nos cidadãos. Para tal, uma estrutura que dinamize esta divulgação junto da sociedade, através dos media, nomeadamente através da seleção do que deve ser divulgado, é da maior importância, trabalhando de perto com os cientistas e com os jornalistas. Cabe a essa estrutura a elaboração de comunicados de imprensa e outros materiais passíveis de serem utilizados pelos órgãos de comunicação social, mas também organizar e mediar, caso necessário, contactos entre cientistas e jornalistas e ajudar a fundamentar a melhor estratégia de posicionamento público em termos científicos, dinamizando também essa ligação com a sociedade.

Para além disso, uma instituição com maior reconhecimento e impacto no seio da sociedade terá, seguramente, maior probabilidade de vir a ser bem-sucedida no contexto de processos de avaliação e financiamento (Raymond, 2013). Este poderá ser também um aspeto particularmente importante para atrair apoio de entidades privadas, como associações, ligas, e outras. Por estas razões, é fundamental o estabelecimento de parcerias e colaborações com entidades com experiência e competências na área da comunicação de ciência, que possam complementar e ajudar a atingir os objetivos da instituição.

\section{Investigação e Inovação}

Comunicar ciência é ainda estar atento a tudo o que rodeia, com especial enfâse no que é novidade. Há que investigar novas formas de comunicação e implementar estratégias inovadoras de formar e treinar comunicadores de ciência. Voltando ao início, uma Faculdade de Medicina, por exemplo, não pode ter como único objetivo a formação de especialistas na área médica. 
Apesar de a comunicação de ciência ser um conteúdo que já vem sendo introduzido nos programas de diversos cursos avançados, em vários ciclos de ensino superior, integrados em 20s e 3 os ciclos oferecidos, não se pode considerar que o trabalho esteja finalizado, principalmente numa área que continua, apesar do franco crescimento ao longo deste século, a dar os primeiros passos. Há, portanto, que desenhar novos programas de formação avançada, dirigidos à comunidade científica e académica, com o objetivo de melhorar o desempenho na comunicação da "sua" ciência, mas também aos comunicadores de ciência, como mediadores da informação científica entre os cientistas e os cidadãos.

Uma estrutura criada com todas estas valências deve ainda criar condições para o desenvolvimento de projetos de investigação nas mais variadas áreas e contextos de comunicação de ciência, seja divulgação de ciência, seja jornalismo de ciência. $O$ enquadramento à escala europeia tem de ser, neste momento, um dos objetivos finais, no âmbito de um alinhamento com aquilo que são as metas definidas para o programa Horizonte 2020, em específico no documento "Strategic opinion for research and innovation in the Horizon 2020 work program 2018-2020". A contribuição para a idealização dos três O's - 'Open innovation, Open science and Openness to the world', no seguimento de estratégias europeias já antigas, de uma ligação cada vez maior entre o mundo académico e científico e a sociedade, e que agora tomam o acrónimo SWAFS - 'Science With And For Society'.

\section{Conclusão}

A comunicação de ciência, e em específico a comunicação de saúde, sempre foram multidisciplinares, mesmo quando debaixo do quase infinito chapéu que é a própria definição do que é ciência e de que ciências se fala quando se fala de ciência. Essa multidisciplinaridade adequa-se ao contexto atual de cada vez maior produção científica e, também, de cada vez maior interligação entre áreas de investigação. Mas falar sobre comunicação científica não é restringi-la apenas ao campo académico, é entender que os dias que correm trazem consigo uma sociedade com uma motivação crescente em dar o seu contributo cívico, em especial quando os temas the dizem respeito. Nesse sentido, a ciência é algo que se mistura inaudivelmente entre o ser humano, assumindo especial destaque, fale-se de saúde ou de tecnologia, de filosofia ou de economia.

A responsabilidade perante a sociedade cabe aos cientistas e às instituições que os acolhem e que eles próprios representam. A área de trabalho é relegada para segundo plano perante a real necessidade, dir-se-ia quase obrigatoriedade, de comunicar o que se faz, da idealização de novas e atrativas formas de o fazer, por forma a demonstrar à sociedade os objetivos da investigação, de que forma esta se insere no quotidiano de cada um e, de uma forma crucial, que passos tem de dar até ser um resultado final. No fundo, a ciência é indelével, sempre existiu, e tem apenas e só de ser totalmente percetível por todos.

\section{Referências}

Araújo, S., Bettencourt-Dias, M., \& Coutinho, A. eds. (2006). Comunicar Ciência: Um Guia Prático para Investigadores. Lisboa: Associação Viver a Ciência.

Barbeiro, L. (2007). Introdução. In L. Barbeiro (Coord.) Comunicação de Ciência, 9-12. Porto: Setepés.

Brownell, S., Price J., \& Steinman, L. (2013a). Science Communication to the General Public: Why We Need to Teach Undergraduate and Graduate Students This Skill as Part of Their Formal Scientific Training. Journal of Undergraduate Neuroscience Education 12.1: E6-E10.

Brownell, S., Price J., \& Steinman, L. (2013b). A Writing-Intensive Course Improves Biology Undergraduates Perception And Confidence Of Their Abilities To Read Scientific Literature And Communicate Science. AJP: Advances In Physiology Education, 37(1), 70-79. doi:10.1152/advan.00138.2012.

Bucchi, M., \& Trench, B. (2016). Science Communication and Science in Society: A Conceptual Review in Ten Keywords. TECNOSCIENZA: Italian Journal of Science \& Technology Studies, 7(2), 151-168.

Burns, W., O'Connor, D., \& Stocklmayer, S. (2003). Science Communication: A Contemporary Definition. Public Understanding of Science, 12(2), 183-202. doi:10.1177/09636625030122004.

Fischhoff, B. (2013). The sciences of science communication. Proceedings Of The National Academy Of Sciences 110 (Supplement_3), 14033-14039. doi:10.1073/pnas.1213273110. 
Garber, M. (2013). “Astro Mad Men: NASA's 1960s Campaign to Win America's Heart". The Atlantic, 31 de julho. https://www.theatlantic.com

Gombrich, E. (2013). A História da Arte - Edição de Bolso. Rio de Janeiro: LTC.

Granado, A., \& Malheiros, J. (2015). Cultura científica em Portugal: Ferramentas para perceber o mundo e para aprender a mudá-lo. Lisboa: Fundação Francisco Manuel dos Santos

Greenwood, M., \& Riordan, D. (2001). "Civic Scientist/Civic Duty". Science Communication, 23(1), $28-40$. doi:10.1177/1075547001023001003.

Gross, L. (2009). "A Broken Trust: Lessons from the Vaccine-Autism Wars". Plos Biology, 7(5), e1000114. doi:10.1371/journal.pbio.1000114.

Gulbrandsen, E. (2016). "From Science in Society to Society in Science". The Norwegian National Research Ethics Comittees, 18 de janeiro. https://www.etikkom.no

Knorr-Cetina, K. (1999). “A Comunicação Na Ciência”. Em Fernando Gil (coord.) A Ciência Tal Qual Se Faz, 375-393. Lisboa: Edições João Sá da Costa.

Leshner, A. (2003). Public Engagement with Science. Science, 299(5609), 977-977. doi:10.1126/science.299.5609.977

Magalhães, R. (2015). A Comunicação Estratégica aplicada à divulgação da Ciência. O caso do Centro de Estudos de Comunicação e Sociedade". Observatorio (OBS*) Journal, 9(4), 051-084.

Miller, J., Scott, E., \& Okamoto, S. (2006). Public Acceptance of Evolution. Science, 313 (5788), $765-766$. doi:10.1126/science.1126746.

Montgomery, S. (2002). The Chicago Guide to Communicating Science. Chicago: The University of Chicago Press.

Nisbet, M. (2010). Framing Science - A New Paradigm in Public Engagement. Em LeeAnn Kahlor, e Patricia A Stout (eds.) Communicating Science: New Agendas in Communication, 40-67. New York: Routledge.

Nisbet, M., \& Dietram A. Scheufele. (2009). What's Next For Science Communication? Promising Directions and Lingering Distractions". American Journal Of Botany, 96(10), 1767- 1778. doi:10.3732/ajb.0900041.

Palmer, S., \& Schibeci, R. (2012). What Conceptions of Science Communication are Espoused by Science Research Funding Bodies?. Public Understanding Of Science, 23(5), 511-527. doi:10.1177/0963662512455295.

Poliakoff, E., \& Webb, T. (2007). What Factors Predict Scientists' Intentions to Participate in Public Engagement of Science Activities?. Science Communication, 29(2), 242-263. doi:10.1177/1075547007308009.

Raymond, J. (2013). New and Old Techniques to Build Your Network, Effectively Tell Your Story, and Obtain Grant Funding. Presentation, American Physiological Society.

Ribeiro, F. (2016). Ritmos da informação/comunicação de ciência dos centros de investigação em Portugal. Cadernos $B A D 2,16-30$.

Shamos, M. (1995). The Myth of Scientific Literacy. Brunswick: Rutgers University Press.

Shipman, Matt. (2014). "Public relations as science communication". JCOM 13(03)C05: 1-6.

Somerville, R., \& Hassol, S. (2011). Communicating the science of climate change. Physics Today, 64(10), 48-53. doi: 10.1063/PT.3.1296

Treise, D., \& Weigold, M. (2002). “Advancing Science Communication”. Science Communication, 23(3), 310-322. doi:10.1177/107554700202300306.

Varner, V. (2014). Scientific Outreach: Toward Effective Public Engagement with Biological Science. Bioscience, 64(4), 333-340. doi:10.1093/biosci/biu021.

Weigold, M. (2001). Communicating Science: A Review of the Literature. Science Communication, 23(2), 164-193. doi:10.1177/1075547001023002005. 


\section{Sobre os Autores}

Paulo Sérgio Nunes dos Santos (MsC) é atualmente doutorando em Ciências da Comunicação na Faculdade de Letras da Universidade de Coimbra, e integra o Laboratório de Comunicação em Saúde da Faculdade de Medicina da Universidade de Coimbra. Nasceu em Coimbra, a 6 de agosto de 1981. É Licenciado em Biologia e possui dois Mestrados em Ecologia e em Ensino de Biologia e Geologia. Foi diretor do Jornal Universitário de Coimbra - A Cabra e da revista Via Latina. Os seus interesses são multidisciplinares, passando pela comunicação de ciência, pelo jornalismo, e em especial o jornalismo de ciência, pela educação e pelo comportamento animal, principalmente em primatas.

António Maria Salvado Coxito Granado (MsC) é professor auxiliar na Faculdade de Ciências Sociais e Humanas da Universidade Nova de Lisboa, tendo lecionado também na Universidade de Coimbra e na Escola Superior de Comunicação Social. Começou o seu percurso como jornalista no Público em 1989, onde se especializou em jornalismo de ciência, tendo ocupado vários cargos até 2010. Desde então, e até 2014, foi editor multimédia na RTP. Nasceu a 10 de janeiro de 1963 em Lisboa. É licenciado em Línguas e Literaturas Modernas, tendo adquirido o grau de Mestre em Jornalismo de Ciência na Universidade de Boston e de Doutor em Ciências da Comunicação na Universidade de Leeds.

Henrique Girão (PhD) é subdiretor para a Investigação e Desenvolvimento, diretor do Laboratório de Microscopia da FMUC, Coordenador do Mestrado em Investigação Biomédica e do Programa de Doutoramento em Ciências da Saúde. No âmbito da sua investigação, tem estudado os mecanismos envolvidos na regulação da comunicação entre células. É responsável pelo Laboratório de Comunicação em Saúde da FMUC. Obteve o grau de Doutor em Ciências Biomédicas, pela Faculdade de Medicina da Universidade de Coimbra (FMUC), em 2006. Na FMUC. Nasceu em 1972, em Coimbra, onde se licenciou em Bioquímica, em 1995. 